\title{
Comparison of steroid hormones in three different preeclamptic models
}

\author{
YE YOUNG SHIN ${ }^{1 *}$, SUNG-MIN AN $^{1 *}$, JEA SIC JEONG ${ }^{1}$, SEUNG YUN YANG ${ }^{1}$, GEUN-SHIK LEE ${ }^{2}$, \\ EUI-JU HONG ${ }^{3}$, EUI-BAE JEUNG ${ }^{4}$, SEUNG CHUL KIM ${ }^{5}$ and BEUM-SOO AN ${ }^{1}$ \\ ${ }^{1}$ Department of Biomaterials Science (BK21 FOUR Program), College of Natural Resources and \\ Life Science/Life and Industry Convergence Research Institute, Pusan National University, Miryang, \\ Gyeongsangnam 50463; ${ }^{2}$ College of Veterinary Medicine, Kangwon National University, Chuncheon, Gangwon 24341; \\ ${ }^{3}$ College of Veterinary Medicine, Chungnam National University, Daejeon 34134; ${ }^{4}$ College of Veterinary Medicine, Chungbuk \\ National University, Cheongju, Chungbuk 28644; ${ }^{5}$ Department of Obstetrics and Gynecology, Biomedical Research Institute, \\ Pusan National University School of Medicine, Busan 49241, Republic of Korea
}

Received May 4, 2020; Accepted October 6, 2020

DOI: $10.3892 / \mathrm{mmr} .2021 .11891$

\begin{abstract}
Preeclampsia (PE) is a complication of pregnancy and is characterized by hypertension and proteinuria, threatening both the mother and the fetus. However, the etiology of PE has not yet been fully understood. Since the imbalance of steroid hormones is associated with the pathogenesis of PE, investigating steroidogenic mechanisms under various $\mathrm{PE}$ conditions is essential to understand the entire spectrum of pregnancy disorders. Therefore, the current study established three PE in vitro and in vivo models, and compared the levels of steroid hormones and steroidogenic enzymes within them. In cellular PE models induced by hypoxia, $\mathrm{N}$-nitro-L-arginine methyl ester hydrocholride (L-NAME) and catechol-o-methyltransferase inhibitor, the levels of steroid hormones, including pregnenolone (P5), progesterone (P4), dehydroepiandrosterone (DHEA) and testosterone tended to decrease during steroidogenesis. Injection of L-NAME in pregnant rats led to a reduction in the levels of estradiol and $\mathrm{P} 4$ through regulation of cholesterol side-chain cleavage enzyme (CYP11A1) and 3 $\beta$-hydroxysteroid dehydrogenase/\$5 4-isomerase type 1 (HSD3B1), whereas rats treated with COMT-I exhibited elevated levels of P5 and DHEA by regulation of the CYP11A1 and aromatase
\end{abstract}

Correspondence to: Professor Beum-Soo An, Department of Biomaterials Science (BK21 FOUR Program), College of Natural Resources and Life Science/Life and Industry Convergence Research Institute, Pusan National University, 50 Cheonghak-ri, Samrangjin-eup, Miryang, Gyeongsangnam 50463, Republic of Korea

E-mail: anbs@pusan.ac.kr

${ }^{*}$ Contributed equally

Key words: preeclampsia, placenta, reduced uterine perfusion pressure, steroid hormones, pregnancy cytochrome P450 (CYP19A1) in the placenta and plasma. The reduced uterine perfusion pressure operation decreased CYP11A1 and increased CYP19A1 expression in placental tissues, whereas steroid hormone levels were not altered. In conclusion, the results of the present study suggest that the induction of PE conditions dysregulates the steroid hormones via regulation of steroidogenic enzymes, depending on specific PE symptoms. These findings can contribute to the development of novel diagnostic and therapeutic modalities for $\mathrm{PE}$, by monitoring and supplying appropriate levels of steroid hormones.

\section{Introduction}

Preeclampsia (PE) is a systemic disorder of pregnancy characterized by the onset of high blood pressure and proteinuria. The clinical manifestations of PE include maternal placental hypoxia, endothelial dysfunction, and imbalance of angiogenic factors (1). PE is a complication in over 5\% of human pregnancies and a leading cause of feto-maternal morbidity and mortality (2).

Although PE ranks as one of the most critical problems in obstetrics, its etiology remains unknown. It is widely accepted that the dysfunction of the placenta due to placental ischemia and hypoxia result in PE (3). Poor placental perfusion caused by failure of the trophoblast cells to replace the spiral artery and imbalanced angiogenic factors increase placental oxidative stress (4). Vascular endothelial growth factor (VEGF) and its receptors play crucial roles in placental angiogenesis. VEGF activates two high-affinity receptor tyrosine kinases, fms-like tyrosine kinase-1 (Flt-1) and fetal liver kinase 1 (Flk-1), to mediate its angiogenic functions. However, binding of the soluble form of Flt-1 (sFlt-1) with VEGF prevents its interaction with endogenous receptors and antagonizes the VEGF-mediated proangiogenic activity. In PE, circulating levels of sFlt-1 levels are high, and thus, the levels of free VEGF are reduced in maternal circulation (5). These conditions lead to fetal growth restriction and low placental weight (6). 
The imbalance in the levels of placental steroid hormones and steroidogenic enzymes is associated with PE pathogenesis. The placenta is the major endocrine organ during pregnancy and secretes several steroid hormones. The multiple steroid hormones are produced by a process called steroidogenesis, which is mediated by steroidogenic enzymes (7). Pregnenolone (P5) is synthesized by cholesterol side-chain cleavage enzyme (CYP11A1) and converted into progesterone (P4) or dehydroepiandrosterone (DHEA) by $3 \beta$-hydroxysteroid dehydrogenase/ $\$ 5$ 4-isomerase type 1 (HSD3B1) or 17 $\alpha$-hydroxylase/17,20-lyase (CYP17A1), respectively. The enzymes including $17 \beta$-dehydrogenase 3 (HSD17B3) and HSD3B1 catalyze the formation of androgens, such as testosterone (T), from DHEA and P5. The final step of steroidogenesis, estrone (E1) and estradiol (E2) biosynthesis, is mediated by the aromatase cytochrome P450 (CYP19A1) and HSD17B. A previous study has shown that the levels of P4 and E2 were downregulated in women with PE, and their levels were modulated by the expression of steroidogenic enzymes in the PE placenta (8).

To study the various aspects of PE, several in vitro and in vivo models have been established (4). One of the well-known models is established by reducing or depleting oxygen in the trophoblast cells and the placenta. Hypoxic conditions tend to inhibit placental invasion and trigger symptoms of PE (9). Inhibition of nitric oxide synthase with N-nitro-L-arginine methyl ester hydrochloride (L-NAME) is also known to mimic PE (10). The combination of a deficiency of nitric oxide (NO) and an increase in peroxynitrite (ONOO-) results in the majority of pathological changes associated with $\mathrm{PE}$, such as high blood pressure, proteinuria, and increased glomerular filtration rate (11). Catechol-o-methyltransferase (COMT) metabolizes 2-hydroxyestradiol to 2-methoxyoestradiol (2-ME), which is involved in the biosynthesis of E2. COMT expression increases with the progression of pregnancy, generating higher levels 2-ME. Pregnant animals deficient in COMT develop PE-like conditions, including proteinuria and placental hypoxia (12). Since PE is caused by a reduction in uterine blood flow due to abnormal trophoblast invasion of the spiral arteries, the reduced uterine perfusion pressure (RUPP) operation generates a suitable PE animal model (1). The RUPP rat model mimics the physiological features of PE, including hypertension and proteinuria, and exhibits increased plasma and placental sFlt-1 and decreased plasma VEGF and placental growth factor $(13,14)$. Although these models have some features of PE, they are not representative of the full spectrum of the human disease because the etiology of PE is most likely multifactorial and may have several forms (15).

Due to the life-threatening risk of PE and the lack of effective treatment options, there have been many attempts to understand the pathogenesis of PE. Recently, many studies addressing the relationship between human PE and steroid hormones have been conducted $(8,10,16)$. However, only steroid hormones in human PE have been studied, and the in vitro and in vivo PE conditions have not been ascertained. To understand the entire steroidogenic machinery and its underlying mechanisms in PE, we established various cellular and animal PE models and compared the levels of steroid hormones during steroidogenesis. Additionally, the levels of steroid hormones in early-stage pregnant women were investigated, who ultimately showed PE symptoms at full term.

\section{Materials and methods}

Cell lines and culture. The BeWo human choriocarcinoma-derived cell line (Korean Cell Line Bank, Seoul, Republic of Korea) were seeded on 6-well plates (7x $10^{5}$ cells/well) in Dulbecco's modified Eagle medium (DMEM; Hyclone) containing 10\% fetal bovine plasma (FBS; Hyclone), $100 \mathrm{IU} / \mathrm{ml}$ of penicillin, and $100 \mu \mathrm{g} / \mathrm{ml}$ of streptomycin and grown at $5 \% \mathrm{CO}_{2}$ at $37^{\circ} \mathrm{C}$. After $24 \mathrm{~h}(\sim 70 \%)$, the cells were placed in a hypoxic chamber (Modular Incubator Chamber; Billups-Rothenberg) to establish a hypoxic model witsh inflow and outflow connectors, and the hypoxic gas consisted of $2 \% \mathrm{O}_{2}, 5 \% \mathrm{CO}_{2}$, and $93 \% \mathrm{~N}_{2}$. Experiments were conducted in an environment at $37^{\circ} \mathrm{C}$ by placing the chambers in an incubator for $24 \mathrm{~h}$. For other in vitro PE models, the cells were treated with L-NAME (100 $\mu \mathrm{M}$; Sigma-Aldrich), Ro 41-0960 (COMT-I; $10 \mu \mathrm{M}$; Sigma-Aldrich), or EtOH as a vehicle control for $24 \mathrm{~h}$ in a $37^{\circ} \mathrm{C}$ incubator. The cell culture medium was extracted for ELISA assays. Restore western blot stripping buffer was obtained from Pierce. All in vitro experiments were performed at least three times in triplicate.

Measurement of cell viability. The BeWo cells were cultured in 24-well plate with density of $3 \times 10^{4}$ cells/well in triplicate and $10 \%$ FBS for $24 \mathrm{~h}$ under various conditions: Vehicle control group, hypoxic condition group, L-NAME $(100 \mu \mathrm{M})$ group, and COMT-I $(10 \mu \mathrm{M})$ group. Then methyl thiazolyl tetrazolium (MTT; $5 \mathrm{mg} / \mathrm{ml}$; Sigma-Aldrich) was added and incubated $4 \mathrm{~h}$, after that we used 100\% DMSO to dissolved the generated formazan crystals. The absorbance of the crystalline at $570 \mathrm{~nm}$ was analyzed with a microplate spectrophotometer (Epoch Bioteck, Instruments). The cell viability of treatment groups were represented as the percentage of viable cells relative to cell viability of the control group.

Animals. Animal studies were approved (approval number; PNU-2017-1452) by the Ethics Committee of Pusan National University (Busan). Twenty-five pregnant female Sprague-Dawley [gestational day (GD) 1] rats were purchased from Central Lab. Animal Inc. (Seoul). The rats were randomly divided into five groups as follows: Control group (CON group; $n=5$ ), L-NAME group ( $n=5)$, COMT-inhibitor group (COMT-I; $n=5)$, Sham group $(n=5)$, and RUPP group $(n=5)$. The rats were housed under standard laboratory conditions with controlled temperature/humidity, a 12:12-h light/dark cycle, and free access to food and water at the Pusan National University Laboratory Animal Resources Center. This facility is accredited by the Korea Food and Drug Administration (AFDA) in accordance with the Laboratory Animal Act (Accredited Unit Number-000231) and AAALAC International according to the National Institutes of Health guidelines (Accredited Unit Number;001525).

Drugs and chemicals. The L-NAME and COMT-I groups were subcutaneously (SC) injected daily with L-NAME $(50 \mathrm{mg} / \mathrm{kg} / \mathrm{day} / 200 \mu \mathrm{l})$ and COMT-I $(2.5 \mathrm{mg} / \mathrm{kg} / \mathrm{day} / 200 \mu \mathrm{l})$ from GD 10 to $17(17,18)$. The CON group was administrated 
$0.9 \%$ saline $(200 \mu \mathrm{l})$. The dosage was adjusted according to changes in body weight (BW). BW, clinical signs, and abnormal behavior were recorded daily throughout the experimental period.

RUPP operation. On GD 14, Sham and RUPP groups were anesthetized with intraperitoneal injection of Zoletil 50 (Virbac, Carros, France) $20 \mathrm{mg} / \mathrm{kg}$ and Rompun (Bayer Korea) $5 \mathrm{mg} / \mathrm{kg}$ solution. A mid-abdominal incision was made as suggested by Fushima et al (3). After the midline incision, a ligature was tied around the aorta above the iliac bifurcation. Since compensatory blood flow to the placenta occurs via an adaptive increase in ovarian blood flow, both right and left uterine arcades are also tied. As complete ligation killed the dams within a day or two of the operation, the aorta and arcades were tied with a nylon thread $0.35 \mathrm{~mm}$ in diameter, followed by removal of the thread to provide a small space for minimal circulation of blood. These procedures reduce uterine blood flow in the gravid rat by $40 \%$. The sham group was operated on similarly, but without ligation. The abdominal incision was then sutured.

Measurement of blood pressure and urinary protein. Blood pressure, especially the systolic blood pressure, is the key criterion when evaluating a pre-eclamptic model (19). From GD 10, the baseline systolic blood pressures were obtained with a blood pressure monitor for mice and rats $\left(\mathrm{CODA}^{\mathrm{TM}}\right.$ Monitor; Kent Scientific Corporation) after the rats were pre-warmed on a warming platform in rat holders. The systolic blood pressure measurements were performed in triplicate, and the mean for each rat was recorded till GD 18. To measure the urinary protein level, rats were placed in metabolic cages on GD 17, and urine was collected over $24 \mathrm{~h}$. The urine was centrifuged at $10,000 \times \mathrm{g}$ for $10 \mathrm{~min}$ at $4^{\circ} \mathrm{C}$ and immediately stored at $-80^{\circ} \mathrm{C}$. Protein levels in the urine were measured using a BCA protein assay kit (Pierce Biotechnology).

Sample collection. On GD 18, the rats were euthanized in gradually filled $\mathrm{CO}_{2}$ gas chamber with a flow rate of $\leq 30 \% \mathrm{CO}_{2}$ of the chamber volume/min. Rats were euthanized in 2018, which corresponds to the period approved by the Ethics Committee of Pusan National University (approval number; PNU-2017-1452). Approximately $1 \mathrm{ml}$ of blood from the inferior vena cava was collected in plastic tubes under aseptic conditions with EDTA as an anticoagulant and centrifuged at $12,000 \mathrm{rpm}$ for $10 \mathrm{~min}$ at $4^{\circ} \mathrm{C}$ to separate the plasma. The plasma was collected and immediately frozen at $-80^{\circ} \mathrm{C}$ until analysis. The placentas were weighed and stored at $-80^{\circ} \mathrm{C}$ for western blot analysis. The kidneys were harvested, fixed in $10 \%$ formalin and embedded in paraffin for histological analysis. The fetuses were isolated to be weighed and counted. The fetal resorption rate was obtained as percent fetal resorption $=$ (number of absorbed fetuses/total number of fetuses) x100, as previously reported (20).

Western blot analysis. Protein samples were prepared with a Pro-prep solution (iNtRON Biotechnology). A total of $20 \mu \mathrm{g}$ protein was analyzed by $10-12 \%$ sodium dodecyl sulfate polyacrylamide gel electrophoresis (SDS-PAGE) and then transferred to nitrocellulose membranes (Daeillab Service
Co, Ltd.). Membranes were subsequently blocked for $1 \mathrm{~h}$ with $5 \%$ skim milk (Difco) in Tris-buffered saline (TBS) with $0.05 \%$ Tween-20(TBS-T).Following blocking,membranes were incubated with hypoxia inducible factor 1 subunit $\alpha$ (HIF1A, 1:500; Santa Cruz Biotechnology, Inc.; cat. no. sc53546), VEGF (1:1,000; Abcam; cat. no. ab46154), sFlt-1 (1:1,000; Abcam; cat. no. ab32152), CYP11A1 (1:1,000; Cell Signaling Technology Inc.; cat. no. 14217), CYP17A1 (1:500; Santa Cruz Biotechnology, Inc.; cat. no. sc46084), HSD3B1 (1:2,000; Santa Cruz Biotechnology, Inc.; cat. no. sc30820), HSD17B3 (1:2,000; Abcam; cat. no. ab55268), and CYP19A1 (1:500; Santa Cruz Biotechnology, Inc.; cat no. sc14244) antibodies overnight at $4^{\circ} \mathrm{C}$, followed by horseradish peroxidase (HRP)-conjugated secondary antibodies (all 1:2,000; Santa Cruz Biotechnology, Inc.; cat. no. sc2313, sc2005, sc2020). Luminol reagent (Bio-Rad) was used to visualize antibody binding. Membranes were subsequently probed with an antibody against $\beta$-actin (1:3,000; Cell Signaling Technology Inc.; cat. no. 8457) as an internal control. For direct comparisons between the concentration levels of different signaling molecules, membranes were stripped and re-probed using Restore western blot stripping buffer as detailed by the manufacturer and re-probed after verifying the absence of residual HRP activity of the membrane. Each blot was scanned using a Bio-Rad ChemiDoc XRS (Bio-Rad), and band intensities were normalized to $\beta$-actin levels.

ELISA assays. Concentrations of P5 (Alpco; cat. no. KA1912), P4 (Cayman Chemical Company; cat. no. 582601), DHEA (Enzo Life Sciences, Inc.; cat. no. 20-DHEHU-E01), T (Cayman Chemical Company; cat. no. 582701), E2 (Cayman Chemical Company; cat. no. 582251), VEGF (R\&D Systems, Inc.; cat. no. RRV00), and sFlt-1 (R\&D Systems, Inc.; cat. no. MVR100) were measured using competitive enzyme immunoassay kits, according to the manufacturers' protocols. The culture medium from BeWo cells, plasma and placenta tissues were added to 96 -well plates. The plate was incubated for $1 \mathrm{~h}$ at room temperature on an orbital shaker. Following incubation at room temperature for 90 min with Ellman's reagent, the optical density was measured spectrophotometrically at $420 \mathrm{~nm}$. The final concentrations were calculated using standard curve analysis.

Histological analysis. Four micrometer paraffin sections of rat kidney tissues were stained with hematoxylin and eosin (Sigma-Aldrich) according to the standard protocol. Tissue images were captured at x400 using a Leica DM 500 microscope (Leica).

Statistical analysis. Results are presented as the mean \pm standard deviation (SD). Data were analyzed for statistical significance using one-way analysis of variance followed by Turkey's multiple comparison test using SPSS version 10.10 for Windows (SPSS, Inc.). $\mathrm{P}<0.05$ was considered to indicate a statistically significant difference.

\section{Results}

Induction of PE conditions in vitro. To generate an in vitro $\mathrm{PE}$ environment, human placenta-derived BeWo cells were 
A

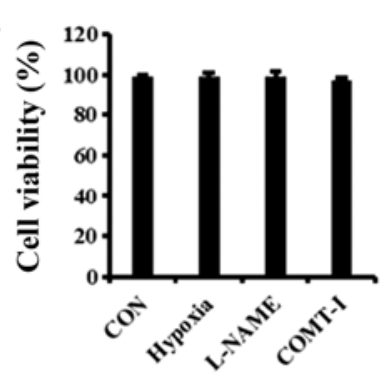

B

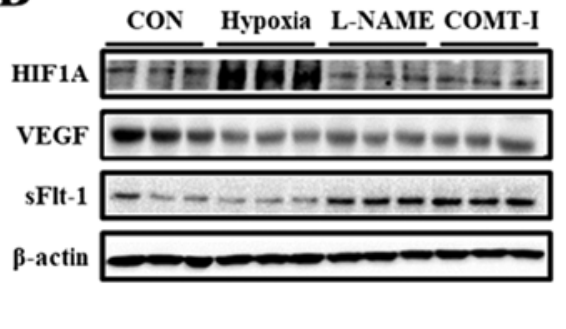

C aCON QHypoxia aL-NAME aCOMT-I

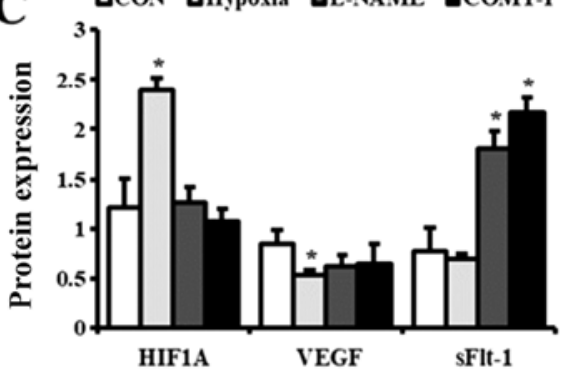

D

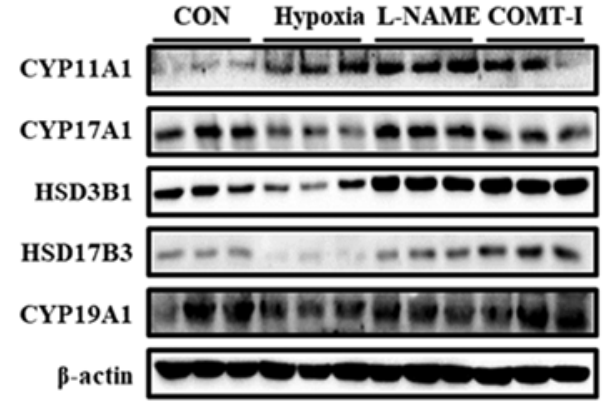

$\mathbf{E}$

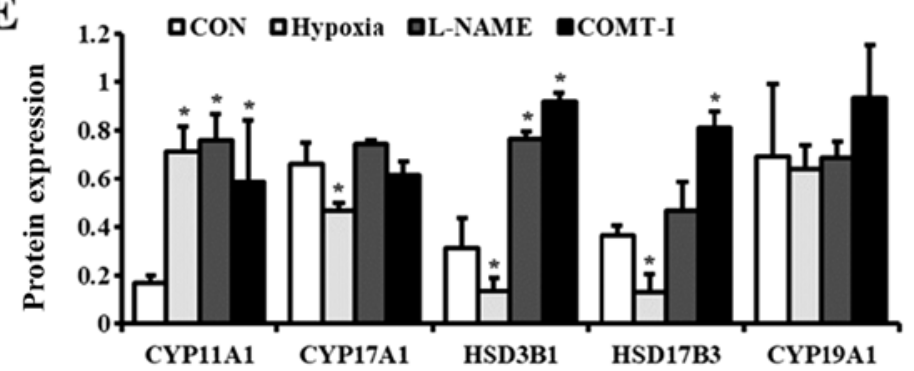

Figure 1. Levels of PE biomarkers and steroidogenic enzymes in in vitro PE models. Cell viability was measured using the MTT assay and demonstrated as a (A) percentage of the CON group. The expression of (B) PE biomarkers in BeWo cells exposed to hypoxia or treated with either L-NAME or COMT-I were analyzed by western blotting. (C) Expression levels of HIF1A, VEGF, and sFlt-1. (D) Representative blot images of steroidogenic enzymes in BeWo cells exposed to hypoxia or treated with either L-NAME or COMT-I. (E) The levels of CYP11A1, CYP17A1, HSD3B1, HSD17B3 and CYP19A1. The individual protein expression level was normalized to that of $\beta$-actin. Data are expressed as the mean $\pm \mathrm{SD}$. ${ }^{*}<0.05$ vs. CON group. CON, control; PE, preeclampsia; L-NAME, N-nitro-L-arginine methyl ester hydrocholride; COMT-I, catechol-o-methyltransferase inhibitor; HIF1A, Hypoxia inducible factor 1 subunit $\alpha$; sFlt-1, soluble form of fetal liver kinase 1; CYP11A1, cholesterol side-chain cleavage enzyme; CYP17A1, 17 $\alpha$-hydroxylase/17,20-lyase; HSD3B1, 3 $\beta$-hydroxysteroid dehydrogenase/\$5 4-isomerase type 1; HSD17B3, 17ß-dehydrogenase 3; CYP19A1, aromatase cytochrome P450.

exposed to hypoxia by incubating the cells in a hypoxic chamber for $24 \mathrm{~h}$. For other models, the cells were treated with L-NAME or COMT-I. The cytotoxicity of all groups was assessed in MTT assay. According to the MTT viability test, hypoxia condition, L-NAME and COMT-I did not exert any effect on the viability of BeWo cells (Fig. 1A). Since HIF1A is a well-known biomarker of hypoxia (21), we examined HIF1A expression to test our experimental conditions by western blot analysis (Fig. 1B and C). HIF1A was dramatically over-expressed in the hypoxic group as expected, whereas it was weakly expressed in other groups. To confirm PE conditions, the expression levels of VEGF and sFlt-1 were examined by western blot analysis (Fig. 1B and C). The expression of VEGF was downregulated by hypoxia, whereas that of sFlt-1 was upregulated by both L-NAME and COMT-I. These results suggest that our in vitro $\mathrm{PE}$ models mimic some of the clinical characteristics observed in PE patients.

Expression of steroidogenic enzymes in in vitro PE models. To elucidate the effect of in vitro PE conditions on the steroidogenesis in placental cells, the expression of steroidogenic enzymes was examined by western blot analysis (Fig. 1D and E). CYP11A1 was upregulated in all groups compared to the control, whereas the levels of CYP19A1 were not altered. Interestingly, the expression of other steroidogenic enzymes was regulated differentially by hypoxia, L-NAME, and COMT-I. Under hypoxic conditions, levels of CYP17A1, HSD3B1, and HSD17B3 were significantly downregulated.
L-NAME increased the protein levels of HSD3B1, whereas COMT-I elevated both HSD3B1 and HSD17B3 expression.

Production of steroid hormones in in vitro PE models. Next, we examined the synthesis of steroid hormones in in vitro PE models. We determined the concentration of steroid hormones, including P5, P4, DHEA, T and E2, by ELISA (Table I). The concentration of steroid hormones tended to decline in every group compared to the CON group. L-NAME and hypoxia significantly decreased the concentration of P5, P4, DHEA, and T. Further, the concentration of DHEA and $\mathrm{T}$ were downregulated by COMT-I. These results suggest that in vitro $\mathrm{PE}$ models, especially those established by hypoxia and L-NAME treatment, affect the production of steroid hormones.

Evaluation of PE characteristics in in vivo PE models. Our next objective was to investigate the association of steroid hormones and PE in animal models. Pregnant rats were treated with L-NAME and COMT-I or underwent RUPP operation. For the biological conditions, symptoms including BW, placental and fetal weight, resorption rate, and urinary protein concentration were evaluated (Table II). All groups showed decreased BW compared to the CON group and the Sham group during the period of treatment. Treatment with L-NAME caused a significant reduction in placental and fetal weight relative to saline solution control. Urinary protein excretion was also higher in the L-NAME group compared to the CON group. Compared to the Sham group, 
Table I. Concentration of steroid hormones in BeWo cells.

\begin{tabular}{|c|c|c|c|c|}
\hline Hormone (ng/ml) & $\mathrm{CON}$ & Hypoxia & L-NAME & COMT-I \\
\hline P5 & $24.1 \pm 3.3$ & $8.3 \pm 1.6^{\mathrm{a}}$ & $14.0 \pm 2.4^{\mathrm{a}}$ & $24.6 \pm 2.4$ \\
\hline P4 & $5.6 \pm 0.1$ & $4.6 \pm 0.2^{\mathrm{a}}$ & $5.0 \pm 0.0^{\mathrm{a}}$ & $5.3 \pm 0.0$ \\
\hline DHEA & $0.8 \pm 0.1$ & $0.1 \pm 0.0^{\mathrm{a}}$ & $0.1 \pm 0.0^{\mathrm{a}}$ & $0.5 \pm 0.1^{\mathrm{a}}$ \\
\hline $\mathrm{T}$ & $0.3 \pm 0.0$ & $0.1 \pm 0.0^{\mathrm{a}}$ & $0.2 \pm 0.0^{\mathrm{a}}$ & $0.2 \pm 0.0^{\mathrm{a}}$ \\
\hline $\mathrm{E} 2$ & $11.9 \pm 2.1$ & $10.7 \pm 1.0$ & $10.4 \pm 1.9$ & $11.0 \pm 0.8$ \\
\hline
\end{tabular}

CON, control; L-NAME, N-nitro-L-arginine methyl ester hydrochloride; COMT-I, catechol-o-methyltransferase inhibitor; P5, pregnenolone; $\mathrm{P} 4$, progesterone; DHEA, dehydroepiandrosterone; T, testosterone; E2, estradiol. Data are presented as the mean $\pm \mathrm{SD}$. ${ }^{\mathrm{a}} \mathrm{P}<0.05$ vs. CON group.

Table II. Characteristics of rats on GD 18.

\begin{tabular}{|c|c|c|c|c|c|}
\hline Characteristic & $\mathrm{CON}$ & L-NAME & COMT-I & Sham & RUPP \\
\hline BW (g) & $312.8 \pm 17.2$ & $279.3 \pm 15.4^{\mathrm{a}}$ & $276.7 \pm 26.4^{\mathrm{a}}$ & $275.0 \pm 20.2$ & $214.8 \pm 20.3^{b}$ \\
\hline Placental weight (g) & $0.5 \pm 0.1$ & $0.4 \pm 0.00^{\mathrm{a}}$ & $0.5 \pm 0.1$ & $0.36 \pm 0.05$ & $0.20 \pm 0.06^{\mathrm{b}}$ \\
\hline Fetal weight (g) & $1.9 \pm 0.2$ & $1.4 \pm 0.3^{\mathrm{a}}$ & $1.8 \pm 0.2$ & $1.32 \pm 0.08$ & $1.36 \pm 0.21$ \\
\hline Fetus (n) & $12.8 \pm 0.8$ & $12.3 \pm 1.5$ & $12.8 \pm 2.9$ & $11.5 \pm 1.5$ & $8.0 \pm 3.39$ \\
\hline Litter (n) & $0.0 \pm 0.0$ & $0.1 \pm 0.4$ & $2.0 \pm 4.9$ & $1.29 \pm 1.91$ & $6.8 \pm 2.38$ \\
\hline Fetal resorption rate $(\%)$ & $0.0 \pm 0.0$ & $1.0 \pm 2.5$ & $16.7 \pm 40.8$ & $13.88 \pm 15.23$ & $89.4 \pm 18.04^{b}$ \\
\hline Urine protein excretion $(\mu \mathrm{g} / \mu \mathrm{l})$ & $4.5 \pm 1.0$ & $6.2 \pm 0.1^{\mathrm{a}}$ & $4.6 \pm 1.1$ & $4.20 \pm 0.74$ & $13.29 \pm 1.29^{\mathrm{b}}$ \\
\hline
\end{tabular}

GD, gestational day; CON, control; L-NAME, N-nitro-L-arginine methyl ester hydrochloride; COMT-I, catechol-o-methyltransferase inhibitor; RUPP, reduced uterine perfusion pressure; BW, body weight. Data are presented as the mean $\pm \mathrm{SD}$. ${ }^{\mathrm{a}} \mathrm{P}<0.05$ vs. CON group, and ${ }^{\mathrm{b}} \mathrm{P}<0.05 \mathrm{vs}$. Sham group.

the RUPP group showed relatively lower placental weight and a higher resorption rate and urinary protein level. However, there were no significant changes in the COMT-I group. The average weight gain was not significantly different for each experimental group (Fig. 2A).

Systolic blood pressures for each experimental group are shown in Fig. 2B. The normal range for systolic blood pressure in rats is around $126 \mathrm{mmHg}$ (22). On GD 14, the systolic blood pressures were significantly elevated in the L-NAME group compared to the CON group $(147.49 \pm 6.68 \mathrm{mmHg}$ vs. $122.79 \pm 8.97 \mathrm{mmHg}, \mathrm{P}<0.05)$, while it recovered from GD 14 to GD 16. On GD 18, both L-NAME and COMT-I groups showed mild hypertension $(135.39 \pm 6.88 \mathrm{mmHg}$ and $130.21 \pm 9.7 \mathrm{mmHg}$ vs. $117.33 \pm 9.63 \mathrm{mmHg}, \mathrm{P}<0.05)$. The systolic blood pressure of the RUPP group was marginally increased compared to the Sham group on GD 18, but the increase was not significant (Fig. 2B).

The effect on renal function observed in the PE rat models was confirmed at the tissue level (Fig. 2C). Histological examination of the kidney revealed that the COMT-I and RUPP groups had endotheliosis involving swelling of the glomerulus and capillary occlusion, whereas it was not observed in the CON and Sham groups. Moreover, hemorrhage was detected in the RUPP group. The concentration of VEGF and sFlt-1 in the plasma of PE models was examined by ELISA (Fig. 3A). The levels of VEGF were significantly lower in the L-NAME and COMT-I groups, whereas there were no significant changes in sFlt-1 levels. To further investigate the placental expression of VEGF and sFlt-1, their levels were analyzed by western blot analysis (Fig. 3C). We found that sFlt-1 was upregulated by COMT-I and VEGF was downregulated by RUPP, whereas L-NAME did not affect the expression of either.

Expression of steroidogenic enzymes in in vivo PE models. As the PE rat models showed features and symptoms similar to human $\mathrm{PE}$, the steroidogenesis process was investigated by western blot analysis (Fig. 4). The CYP11A1 protein levels were decreased in all groups compared with $\mathrm{CON}$ and Sham groups. The expression of HSD3B1 was significantly upregulated (up to 1.8-fold) by L-NAME. The levels of CYP19A1 were elevated by COMT-I and RUPP. CYP17A1 and HSD17B3 levels were unaltered in all in vivo PE models.

Concentration of steroid hormones in in vivo PE models. Based on the regulation of steroidogenesis in the placenta of rats, the concentration of steroid hormones was determined by ELISA (Tables III and IV). L-NAME reduced the levels of E2 2-fold, while COMT-I significantly increased concentration of P5 and DHEA in the plasma (Table III). The concentration of other steroid hormones were not significantly altered. The placental levels of steroid hormones were different compared to their levels in the plasma (Table IV). The levels of P4 in the placenta of the L-NAME group were 2-fold lower than 

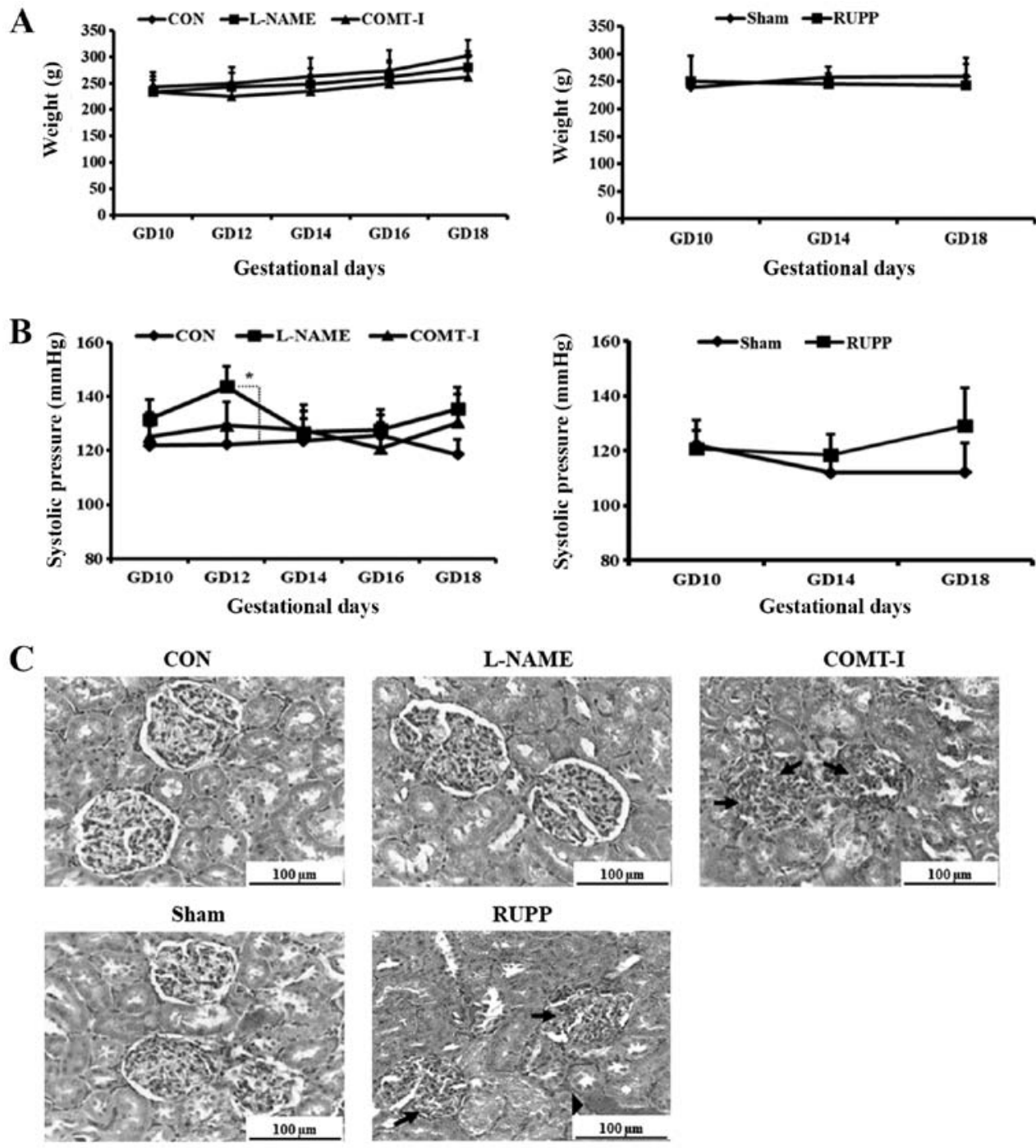

Figure 2. Analysis of systolic blood pressure and kidney function. (A and B) The average weight and the systolic blood pressure from GD10 to GD18 in L-NAME, COMT-I and RUPP groups was measured and compared with the CON or Sham group. Blood pressure was measured in triplicate, and the mean for each rat was recorded until GD 18. (C) The kidneys were stained with hematoxylin and eosin. Occlusion of the capillary loops with an increase in cellularity was observed in the enlarged glomerulus (arrows) in the COMT-I and RUPP groups. Hemorrhage (triangle) was detected in the RUPP group. Data are expressed as the mean $\pm \mathrm{SD}$. ${ }^{*} \mathrm{P}<0.05$ vs. CON group. L-NAME, N-nitro-L-arginine methyl ester hydrocholride; COMT-I, catechol-o-methyltransferase inhibitor; RUPP, reduced uterine perfusion pressure; CON, control; GD, gestational day.

those in the CON group. Other placental steroid hormones were not significantly altered. The RUPP operation did not affect the levels of any steroid hormones in both plasma and placenta.

\section{Discussion}

It is well-known that hormonal changes are associated with the pathology of PE. Therefore, the assessment of steroid hormone levels in the blood has been the focus of intense study to find potential biomarkers to predict PE (23). Multiple PE models have been developed to mimic the physiological conditions of PE. However, these models are limited in their ability to represent a disease with complicated interactions and the effects on PE-related tissues, and thus single model can't reproduce all the clinical aspects of the PE syndrome (9). Considering this, we established three in vitro and in vivo PE models via inducing hypoxia and via L-NAME, and COMT-1 treatments, and evaluated PE-related biomarkers and pathological characteristics. In addition, we examined how the three PE models affect steroidogenesis in the placenta and hormone levels in the blood.

For our in vitro models, placental cells were exposed to hypoxic conditions or treated with L-NAME and COMT-I. The expression of HIF1A, VEGF, and sFlt-1 in the placenta are predictive biomarkers of PE (24). HIF1 acts as a central regulator of oxygen homeostasis in most mammalian cells. In placenta, HIF1 is essential for placental development regulating several genes that control cell growth, differentiation, and metabolism including proangiogenic factors (25). HIF1A expression can be increased by the inhibition of NOS and COMT activity in the placenta, and consequently result in ubiquitous placental tissue damage $(26,27)$. One study demonstrated that the levels of mRNA and protein expression of placental HIF1A were significantly increased in rats administered $0.5 \mathrm{mg} / \mathrm{ml}$ of L-NAME via drinking water from 
A

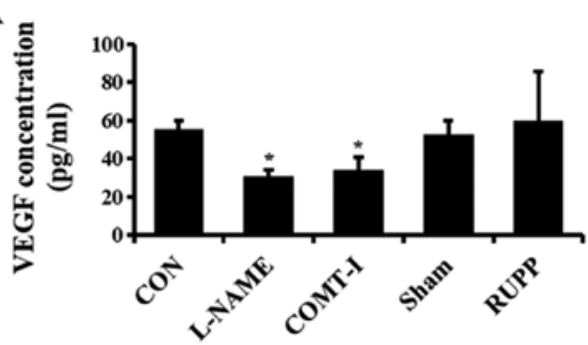

C

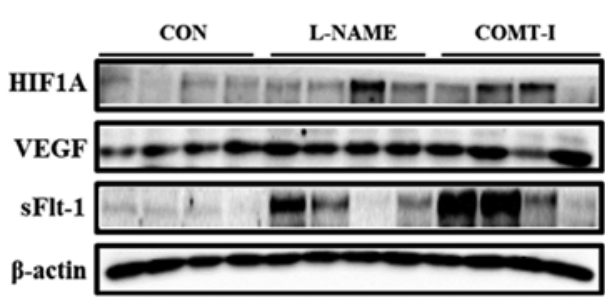

B
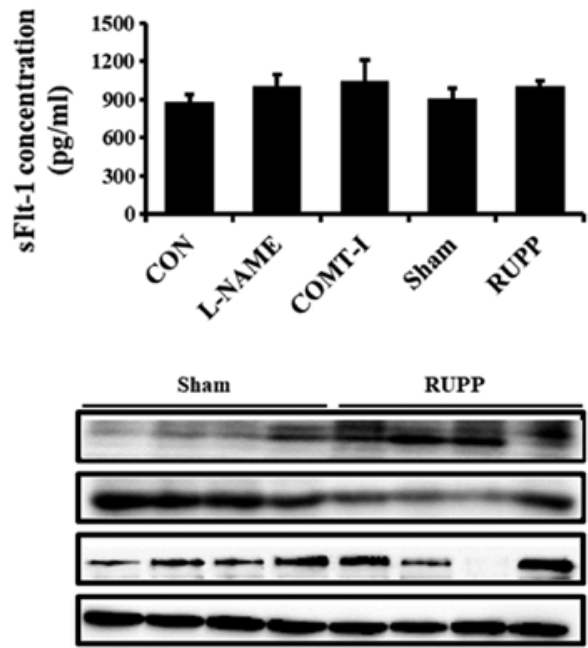

$\mathbf{E}$

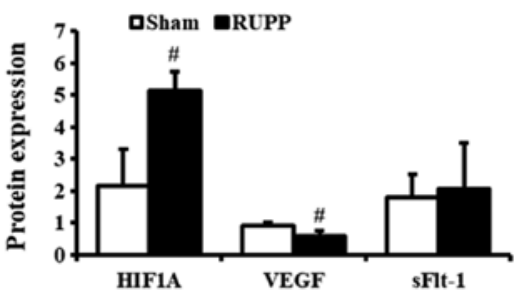

Figure 3. Effects of angiogenic factors on in vivo PE models. (A and B) The plasma concentrations of VEGF and sFlt-1 in L-NAME, COMT-I and RUPP groups were analyzed by ELISA and compared with the CON or Sham group. (C) The expression of PE biomarkers in the placenta of in vivo rat models was analyzed by western blot analysis. (D and E) Expression levels of HIF1A, VEGF and sFlt-1 in the L-NAME, COMT-I, and RUPP groups. Data are expressed as the mean \pm SD. ${ }^{*} \mathrm{P}<0.05$ vs. CON group; ${ }^{\#} \mathrm{P}<0.05$ vs. Sham group. sFlt-1, soluble form of fetal liver kinase 1; L-NAME, N-nitro-L-arginine methyl ester hydrocholride; COMT-I, catechol-o-methyltransferase inhibitor; RUPP, reduced uterine perfusion pressure; CON, control; PE, Preeclampsia; HIF1 $\alpha$, hypoxia inducible factor 1 subunit $\alpha$.
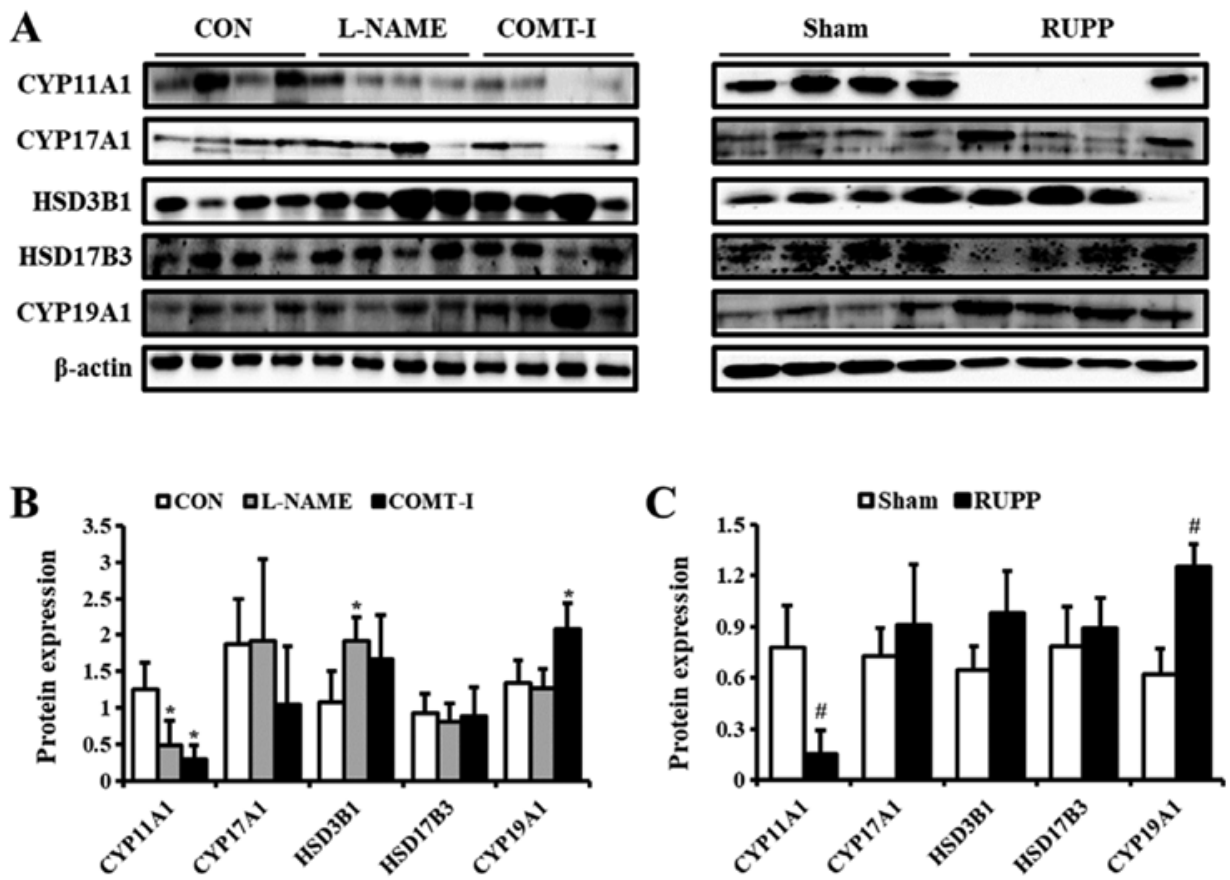

Figure 4. Levels of steroidogenic enzymes in in vivo PE models. (A) The expression of steroidogenic enzymes in the L-NAME, COMT-I, and RUPP groups was analyzed by western blot analysis. (B and C) The levels of CYP11A1, CYP17A1, HSD3B1, HSD17B3 and CYP19A1. The individual protein expression was normalized to that of $\beta$-actin. Data are expressed as the mean $\pm \mathrm{SD}$. "P<0.05 vs. CON group; ${ }^{*} \mathrm{P}<0.05$ vs. Sham group. L-NAME, N-nitro-L-arginine methyl ester hydrocholride; COMT-I, catechol-o-methyltransferase inhibitor; RUPP, reduced uterine perfusion pressure; CYP11A1, cholesterol side-chain cleavage

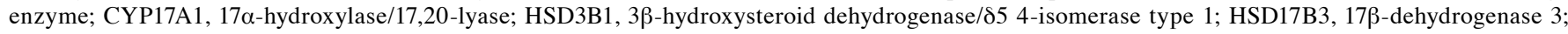
CYP19A1, aromatase cytochrome P450; CON, control. 
Table III. Plasma concentration of steroid hormones in vivo PE models.

\begin{tabular}{|c|c|c|c|c|c|}
\hline Hormone (ng/ml) & $\mathrm{CON}$ & L-NAME & COMT-I & Sham & RUPP \\
\hline P5 & $1.6 \pm 0.1$ & $1.6 \pm 0.4$ & $3.4 \pm 0.5^{\mathrm{a}}$ & $1.4 \pm 0.8$ & $1.3 \pm 0.4$ \\
\hline P4 & $42.2 \pm 7.9$ & $37.8 \pm 4.4$ & $45.8 \pm 6.4$ & $38.8 \pm 13.0$ & $34.9 \pm 7.8$ \\
\hline DHEA & $0.5 \pm 0.1$ & $0.5 \pm 0.10$ & $0.9 \pm 0.1^{\mathrm{a}}$ & $0.6 \pm 0.2$ & $0.4 \pm 0.1$ \\
\hline $\mathrm{T}$ & $8.7 \pm 2.3$ & $8.9 \pm 2.1$ & $9.6 \pm 1.5$ & $8.6 \pm 4.3$ & $7.6 \pm 4.7$ \\
\hline E2 & $0.8 \pm 0.2$ & $0.4 \pm 0.0^{\mathrm{a}}$ & $0.7 \pm 0.1$ & $0.7 \pm 0.4$ & $0.8 \pm 0.3$ \\
\hline
\end{tabular}

PE, preeclampsia; CON, control; L-NAME, N-nitro-L-arginine methyl ester hydrochloride; COMT-I, catechol-o-methyltransferase inhibitor; RUPP, reduced uterine perfusion pressure; P5, pregnenolone; P4, progesterone; DHEA, dehydroepiandrosterone; T, testosterone; E2, estradiol. Data are presented as the mean $\pm \mathrm{SD}$. ${ }^{\mathrm{a}} \mathrm{P}<0.05$ vs. CON group.

Table IV. Placental concentration of steroid hormones in vivo PE models.

\begin{tabular}{|c|c|c|c|c|c|}
\hline Hormone & $\mathrm{CON}$ & L-NAME & COMT-I & Sham & RUPP \\
\hline P5 (ng/ml) & $30.0 \pm 0.5$ & $30.3 \pm 2.0$ & $29.7 \pm 2.7$ & $28.6 \pm 0.8$ & $25.9 \pm 2.1$ \\
\hline P4 (ng/ml) & $0.2 \pm 0.0$ & $0.1 \pm 0.0^{\mathrm{a}}$ & $0.3 \pm 0.0$ & $0.2 \pm 0.1$ & $0.3 \pm 0.1$ \\
\hline DHEA (ng/ml) & $0.5 \pm 0.0$ & $0.5 \pm 0.0$ & $0.6 \pm 0.1$ & $0.4 \pm 0.1$ & $0.3 \pm 0.1$ \\
\hline $\mathrm{T}(\mathrm{ng} / \mathrm{ml})$ & $4.1 \pm 0.5$ & $4.5 \pm 0.6$ & $4.7 \pm 0.8$ & $4.0 \pm 0.5$ & $4.6 \pm 1.9$ \\
\hline E2 (ng/ml) & $5.0 \pm 0.6$ & $4.5 \pm 0.6$ & $4.5 \pm 0.6$ & $4.5 \pm 0.8$ & $4.5 \pm 0.2$ \\
\hline
\end{tabular}

PE, preeclampsia; CON, control; L-NAME, N-nitro-L-arginine methyl ester hydrochloride; COMT-I, catechol-o-methyltransferase inhibitor; RUPP, reduced uterine perfusion pressure; P5, pregnenolone; P4, progesterone; DHEA, dehydroepiandrosterone; T, testosterone; E2, estradiol. Data are presented as the mean $\pm \mathrm{SD}$. ${ }^{\mathrm{a}} \mathrm{P}<0.05$ vs. CON group.

GD 6 to GD 10. Also, in another study, dramatically increased placental HIF1 protein level is observed in $\mathrm{KO}$ mice with a deficiency in COMT $(28,29)$. However, in our study, hypoxia increased the levels of HIF1A, while L-NAME and COMT-I did not. These results were also seen in our in vivo models, where L-NAME and COMT-I did not alter levels of HIF1A while RUPP enhanced HIF1A in the placenta. These distinct results may be due to differences in treatment period and dosage compared to previous studies.

VEGF, known as a target gene of HIF-1A, initiate angiogenesis and induce placental hypoxia (30). The VEGF/sFlt-1 pathway may be induced by hypoxia through HIF-dependent and HIF-independent pathways and the relative overproduction of sFlt-1 than VEGF by placental tissue is one of the symptoms of PE $(31,32)$. In our in vitro study, the VEGF was decreased by hypoxic condition, but not by L-NAME and COMT-I, which suggest that the process of angiogenesis was regulated by hypoxia. However, both L-NAME and COMT-I upregulated the expression of sFlt-1, meaning that the treatments also inhibit angiogenesis in a different way from hypoxia in placental cell. Collectively, our COMT-I- and VEGF-induced PE models showed higher levels of sFlt-1 than VEGF and we suggest that these results may be mediated by HIF1A-independent pathway in vitro and in vivo models.

The pathological characteristics, including placental and fetal weight, proteinuria, hypertension, and glomerular endotheliosis, were analyzed in the in vivo models. Lower venous capacitance and compliance have been suggested to increase blood pressure and protein excretion in PE (33). Fetal growth restriction is a result of reduced placental blood flow due to altered placental vasculature caused by angiogenic factors and impaired development of the placenta (34). Consistent with previous findings, the RUPP operation caused a significant reduction in fetal and placental weights and fetal death. Although blood pressure elevation was not significant in the RUPP group, proteinuria was observed due to endotheliosis and massive bleeding, which had spread beyond the glomerulus. L-NAME administration in pregnant rats led to hypertension, proteinuria, and fetal growth restriction. The rats treated with COMT-I showed hypertension, glomerular endotheliosis, and glomerular injury. The L-NAME and COMT-1 groups showed a lower concentration of VEGF in the blood. These results indicate that the three different PE models showed more than one pathological characteristic of PE, although the underlying mechanisms are different. Among the models, the L-NAME group was closest to representing PE symptoms, because hypertension and proteinuria are considered as the most critical factors for $\mathrm{PE}$.

To investigate the effect of PE conditions on the production of steroid hormones, we examined their levels in the culture-supernatant of placental cells. The levels of steroid hormones, including P5, P4, DHEA, and T, tended to decrease in the hypoxia and L-NAME groups, while the COMT-I group showed lower DHEA and T levels. We also evaluated the expression of steroidogenesis-related enzymes in the placental cells. Although the enzymes were regulated differentially by individual PE models, CYP11A1 was upregulated in all in vitro $\mathrm{PE}$ models. CYP11A1 is a rate-limiting enzyme for 

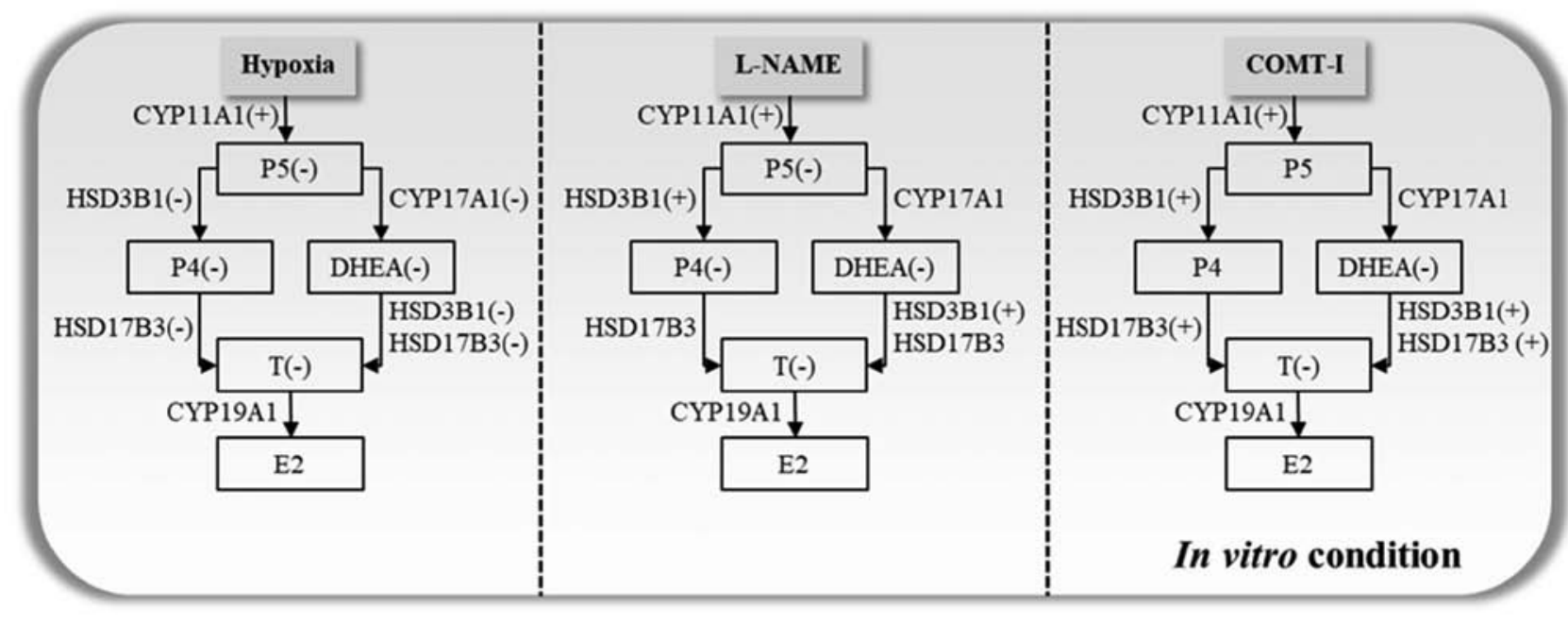

In vitro condition

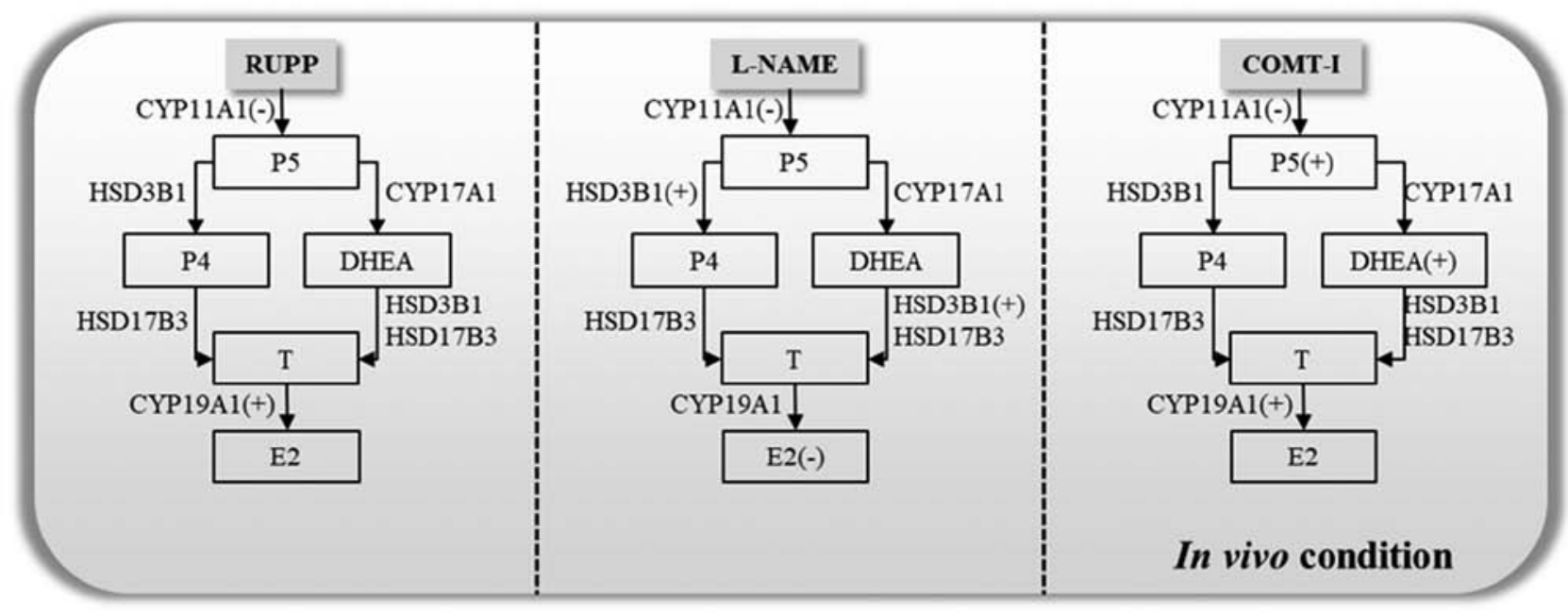

Figure 5. Scheme illustrating how various symptoms of PE regulate steroidogenesis in vitro and in vivo based on the changes observed in plasma. L-NAME, N-nitro-L-arginine methyl ester hydrocholride; COMT-I, catechol-o-methyltransferase inhibitor; RUPP, reduced uterine perfusion pressure; CYP11A1, cholesterol side-chain cleavage enzyme; CYP17A1, 17 $\alpha$-hydroxylase/17,20-lyase; HSD3B1, 3 $\beta$-hydroxysteroid dehydrogenase/ $\delta 5$ 4-isomerase type 1; HSD17B3, 17ß-dehydrogenase 3; CYP19A1, aromatase cytochrome P450; E2, estradiol; P4, progesterone; P5, pregnenolone; T, testosterone; DHEA, dehydroepiandrosterone.

the production of P5 that is the precursor hormone of all other steroid hormones. This could be a compensatory regulation because P5 and its downstream steroid hormones, such as P4, DHEA, and T, were downregulated. These results indicate that the symptoms of PE are closely linked with the levels of steroid hormones and different pathological symptoms of PE may modulate the process of steroidogenesis in distinct ways in the placental cells. The regulation of steroidogenesis in the cellular models of PE in this study were different from the human placenta. It has been demonstrated that P4 and E2 were decreased, whereas other steroid hormones were not altered in human PE (10,35). The steroidogenic process in the human placenta is often influenced by the fetal endocrine system. The fetal adrenal cortex mostly synthesizes byproducts, DHEA and DHEA-S, which are delivered to the placenta for the production of androgens and E2 (36). Since feto-placental communication is absent in in vitro models, there are bound to be differences between the in vitro data and the results from animal and human studies.

We next investigated the steroidogenic process under in vivo $\mathrm{PE}$ conditions. Because of the different species and $\mathrm{PE}$ conditions, the regulation of steroid hormones in our in vivo PE models is different from that seen in in vitro models and human with PE. Both rat and human placentas are anatomically classified as discoid and hemochorial type. However, there are differences between rats and humans in terms of the histological structure of the placenta, the feto-maternal interface, and the function of the yolk sac (37).

We found that lower placental blood flow induced by RUPP affects the levels of CYP11A1 and CYP19A1 in placental tissues, which may, in turn, affect the production of P5 and E2. However, the quantity of steroid hormones produced was not significantly altered in our study. L-NAME treatment in pregnant rats led to a downregulation of VEGF, lower placental weight, and fetal growth restriction. Additionally, L-NAME decreased plasma E2 levels, although the levels were not regulated in the placenta. These results indicated that the plasma E2 was not directly regulated by placenta. It was possibly modulated by fetus or extra-placental tissues in mother. Indeed, the levels of $\mathrm{E} 2$ is regulated by complicated maternal-placental-fetal unit (38). L-NAME inhibits the production of NO which is associated with E2 production (39). Previous studies have 
demonstrated that fetal- and placental-VEGF were stimulated by $\mathrm{E} 2$, indicating that the decrease in $\mathrm{E} 2$ production obstructs angiogenesis and causes oxidative stress in the placenta $(40,41)$. The damaged placenta by oxidative stress can affect the growth and function of the placenta, including the production of steroid hormones. Further, oxidative stress plays a role as an anti-angiogenic factor in blood vessels.

As we mentioned, the rats deficient in COMT showed lower levels of VEGF and elevated sFlt-1. It has been reported that COMT deficiency induces defects in both angiogenesis and the placenta by dysfunction of angiogenic factors (42). Our results from the COMT-I model showed that disrupting angiogenesis in the placenta may lead to the overproduction of P5 and DHEA through the regulation of CYP11A1 and CYP19A1. It is widely accepted that DHEA is associated with vascular angiogenesis $(43,44)$.

In conclusion, we compared steroid hormones and steroidogenic enzymes in three different in vitro and in vivo $\mathrm{PE}$ models. The steroid hormones were dynamically dysregulated depending on the symptoms of PE. The overall in vitro and in vivo results are illustrated in Fig. 5. Our findings provide new insights into the correlation of steroid hormones with PE and may contribute to the development of new diagnostic and therapeutic methodologies for PE.

\section{Acknowledgements}

Not applicable.

\section{Funding}

The present study was supported by a 2-year research grant from Pusan National University (grant no. 201912470002) and also partially supported by the BK21 FOUR Program through the National Research Foundation (NRF) of Korea funded by the Ministry of Education, Korea.

\section{Availability of data and materials}

The datasets used and/or analyzed during the present study are available from the corresponding author on reasonable request.

\section{Authors' contributions}

YYS, SMA and BSA designed the experiments and wrote the manuscript. YYS, SMA, JSJ, SYY and SCK performed the experiments and analyzed the data. GSL, EJH, EBJ and BSA analyzed the data and revised the manuscript. All authors read and approved the final manuscript.

\section{Ethics approval and informed consent}

The animal protocol used in this study was reviewed and approved based on ethical procedures and scientific care by the Pusan National University-Institutional Animal Care and Use Committee (PNU-IACUC) (Approval no. PNU-2017-1452).

\section{Patient consent for publication}

Not applicable.

\section{Competing interests}

The authors declare that they have no competing interests.

\section{References}

1. Li J, LaMarca B and Reckelhoff JF: A model of preeclampsia in rats: The reduced uterine perfusion pressure (RUPP) model. Am J Physiol Heart Circ Physiol 303: H1-H8, 2012.

2. Dragun D and Haase-Fielitz A: Low catechol-O-methyltransferase and 2-methoxyestradiol in preeclampsia: More than a unifying hypothesis. Nephrol Dial Transplant 24: 31-32, 2009.

3. Fushima T, Sekimoto A, Minato T, Ito T, Oe Y, Kisu K, Sato E, Funamoto K, Hayase T, Kimura Y, et al: Reduced uterine perfusion pressure (RUPP) model of preeclampsia in mice. PLoS One 11: e0155426, 2016.

4. Talebianpoor MS and Mirkhani H: The effect of tempol administration on the aortic contractile responses in rat preeclampsia model. ISRN Pharmacol 2012, 187208, 2012.

5. AbdAlla S, Lother H, el Massiery A and Quitterer U: Increased AT(1) receptor heterodimers in preeclampsia mediate enhanced angiotensin II responsiveness. Nat Med 7: 1003-1009, 2001.

6. McKinney D, Boyd H, Langager A, Oswald M, Pfister A and Warshak CR: The impact of fetal growth restriction on latency in the setting of expectant management of preeclampsia. Am J Obstet Gynecol 214: 395.e1-e7, 2016.

7. Sato K, Iemitsu M, Matsutani K, Kurihara T, Hamaoka T and Fujita S: Resistance training restores muscle sex steroid hormone steroidogenesis in older men. FASEB J 28: 1891-1897, 2014.

8. Shin YY, Jeong JS, Park MN, Lee JE, An SM, Cho WS, Kim SC, An BS and Lee KS: Regulation of steroid hormones in the placenta and serum of women with preeclampsia. Mol Med Rep 17: 2681-2688, 2018.

9. Pennington KA, Schlitt JM, Jackson DL, Schulz LC and Schust DJ: Preeclampsia: Multiple approaches for a multifactorial disease. Dis Model Mech 5: 9-18, 2012.

10. Hertig A, Liere P, Chabbert-Buffet N, Fort J, Pianos A, Eychenne B, Cambourg A, Schumacher M, Berkane N, Lefevre G, et al: Steroid profiling in preeclamptic women: Evidence for aromatase deficiency. Am J Obstet Gynecol 203: 477.e1-e9, 2010.

11. Lowe DT: Nitric oxide dysfunction in the pathophysiology of preeclampsia. Nitric Oxide 4: 441-458, 2000.

12. Kanasaki K, Palmsten K, Sugimoto H, Ahmad S, Hamano Y, Xie L, Parry S, Augustin HG, Gattone VH, Folkman J, et al: Deficiency in catechol-O-methyltransferase and 2-methoxyoestradiol is associated with pre-eclampsia. Nature 453: 1117-1121, 2008.

13. Suzuki H, Ohkuchi A, Shirasuna K, Takahashi H, Usui R and Matsubara S: Animal models of preeclampsia: Insight into possible biomarker candidates for predicting preeclampsia. Med J Obstet Gynecol 2: 1031, 2014.

14. Gilbert JS, Babcock SA and Granger JP: Hypertension produced by reduced uterine perfusion in pregnant rats is associated with increased soluble fms-like tyrosine kinase-1 expression. Hypertension 50: 1142-1147, 2007.

15. Kumasawa K: Animal models in preeclampsia. In: Preeclampsia. Springer Nature Singapore, pp141-155, 2018.

16. Zeisler H, Jirecek S, Hohlagschwandtner M, Knofler M, Tempfer $\mathrm{C}$ and Livingston JC: Concentrations of estrogens in patients with preeclampsia. Wien Klin Wochenschr 114: 458-461, 2002.

17. Sun MN and Zi Y: Effects of preeclampsia-like symptoms at early gestational stage on feto-placental outcomes in a mouse model. Chin Med J (Engl) 123: 707-712, 2010.

18. Yang H, Ahn C and Jeung EB: Differential expression of calcium transport genes caused by COMT inhibition in the duodenum, kidney and placenta of pregnant mice. Mol Cell Endocrinol 401: 45-55, 2015.

19. Nathan HL, Duhig K, Hezelgrave NL, Chappell LC and Shennan AH: Blood pressure measurement in pregnancy. Obstetr Gynaecol 17: 91-98, 2015.

20. Spradley FT, Tan AY, Joo WS, Daniels G, Kussie P, Karumanchi SA and Granger JP: Placental growth factor administration abolishes placental ischemia-induced hypertension. Hypertension 67: 740-747, 2016. 
21. Berg A, Fasmer KE, Mauland KK, Ytre-Hauge S, Hoivik EA, Husby JA, Tangen IL, Trovik J, Halle MK, Woie K, et al: Tissue and imaging biomarkers for hypoxia predict poor outcome in endometrial cancer. Oncotarget 7: 69844-69856, 2016.

22. Yasujima M, Abe K, Kohzuki M, Tanno M, Kasai Y, Sato M, Omata K, Kudo K, Takeuchi K, Hiwatari M, et al: Effect of atrial natriuretic factor on angiotensin II-induced hypertension in rats. Hypertension 8: 748-753, 1986.

23. Costa RA, Hoshida MS, Alves EA, Zugaib M and Francisco RP Preeclampsia and superimposed preeclampsia: The same disease? The role of angiogenic biomarkers. Hypertens Pregnancy 35: 139-149, 2016.

24. Tal R: The role of hypoxia and hypoxia-inducible factor-1alpha in preeclampsia pathogenesis. Biol Reprod 87: 134, 2012.

25. Gaspar JM and Velloso LA: Hypoxia inducible factor as a central regulator of metabolism-implications for the development of obesity. Front Neurosci 12: 813, 2018

26. Olson $\mathrm{N}$ and Van Der Vliet A: Interactions between nitric oxide and hypoxia-inducible factor signaling pathways in inflammatory disease. Nitric Oxide 25: 125-137, 2011

27. Arendt KW and Garovic VD: Association of deficiencies of catechol-O-methyltransferase and 2-methoxyestradiol with preeclampsia. Expert Rev Obstetr Gynecol 4: 379-381, 2009.

28. Zheng L, Huang J, Su Y, Wang F, Kong H and Xin H: Vitexin ameliorates preeclampsia phenotypes by inhibiting TFPI-2 and HIF-1 $\alpha$ /VEGF in al-NAME induced rat model. Drug Dev Res 80: 1120-1127, 2019.

29. Kulkarni K: HIF-1 alpha: A master regulator of trophoblast differentiation and placental development. Browse Theses Dissertations: 943, 2009. https://corescholar.libraries.wright. edu/etd_all/943. Accessed September 3, 2020.

30. Saravani M, Rokni M, Mehrbani M, Amirkhosravi A, Faramarz S, Fatemi I, Esmaeili Tarzi M and Nematollahi MH: The evaluation of VEGF and HIF-1 $\alpha$ gene polymorphisms and multiple sclerosis susceptibility. J Gene Med 21: e3132, 2019.

31. Mizukami Y, Li J, Zhang X, Zimmer MA, Iliopoulos O and Chung DC: Hypoxia-inducible factor-1-independent regulation of vascular endothelial growth factor by hypoxia in colon cancer. Cancer Res 64: 1765-1772, 2004

32. Pant V, Yadav BK and Sharma J: A cross sectional study to assess the sFlt-1:PlGF ratio in pregnant women with and without preeclampsia. BMC Pregnancy Childbirth 19: 266, 2019.

33. Schrier RW (ed.): Renal and Electrolyte Disorders. Lippincott Williams \& Wilkins, Philadelphia, PA, 2010.
34. Banek CT, Bauer AJ, Gingery A and Gilbert JS: Timing of ischemic insult alters fetal growth trajectory, maternal angiogenic balance, and markers of renal oxidative stress in the pregnant rat. Am J Physiol Regul Integr Comp Physiol 303: R658-R664, 2012.

35. Salari S, Eskandari M, Nanbakhsh F and Dabiri A: Evaluation of androgen and progesterone levels in women with preeclampsia. Iranian J Med Sci 30: 186-189, 2015.

36. Kaludjerovic J and Ward WE: The interplay between estrogen and fetal adrenal cortex. J Nutr Metab 2012: 837901, 2012.

37. Furukawa S, Tsuji N and Sugiyama A: Morphology and physiology of rat placenta for toxicological evaluation. J Toxicol Pathol 32: 1-17, 2019.

38. Buster JE: Gestational changes in steroid hormone biosynthesis, secretion, metabolism, and action. Clin Perinatol 10: 527-552, 1983.

39. Hu XQ, Song R and Zhang L: Effect of oxidative stress on the estrogen-NOS-NO-KCa channel pathway in uteroplacental dysfunction: Its implication in pregnancy complications. Oxid Med Cell Longev 2019: 9194269, 2019.

40. Vonnahme KA, Wilson ME and Ford SP: Relationship between placental vascular endothelial growth factor expression and placental/endometrial vascularity in the pig. Biol Reprod 64: $1821-1825,2001$.

41. Maliqueo M, Echiburú B and Crisosto N: Sex steroids modulate uterine-placental vasculature: Implications for obstetrics and neonatal outcomes. Front Physiol 7: 152, 2016.

42. Kanasaki $\mathrm{K}$ and Kanasaki $\mathrm{M}$ : Angiogenic defects in preeclampsia: What is known, and how are such defects relevant to preeclampsia pathogenesis? Hypertension Res Pregnancy 1: 57-65, 2013.

43. Liu D, Iruthayanathan M, Hosman LL, Wang Y, Yang L, Wang Y and Dillon JS: Dehydroepiandrosterone stimulates endothelial proliferation and angiogenesis through extracellular signal-regulated kinase 1/2-mediated mechanisms. Endocrinology 149: 889-898, 2007.

44. Barnabas O, Wang $\mathrm{H}$ and Gao XM: Role of estrogen in angiogenesis in cardiovascular diseases. J Geriatr Cardiol 10: 377-382, 2013.

This work is licensed under a Creative Commons Attribution-NonCommercial-NoDerivatives 4.0 International (CC BY-NC-ND 4.0) License. 